\title{
Standards of Practice in Transarterial Radioembolization
}

\author{
Andreas H. Mahnken • Carlo Spreafico • \\ Geert Maleux • Thomas Helmberger • \\ Tobias F. Jakobs
}

Received: 12 October 2012/ Accepted: 11 February 2013/Published online: 20 March 2013

(C) Springer Science+Business Media New York and the Cardiovascular and Interventional Radiological Society of Europe (CIRSE) 2013

\section{Introduction}

\section{Rationale of Radioembolization}

Primary cancer to the liver is the second most frequent cause of cancer death worldwide. There are 750,000 new cases of liver cancer worldwide [1], and metastatic liver disease is the most common cause of cancer death. Only about $25 \%$ of patients with primary or secondary liver cancer are eligible for surgery, with a roughly $50 \% 5$-year overall survival in metastatic colorectal cancer (CRC) and little more than 20 months' median overall survival with systemic chemotherapy alone [2]. Thus, interventional techniques evolved as a first-line treatment in primary liver tumors and are

\footnotetext{
A. H. Mahnken ( $\square)$

Department of Diagnostic and Interventional Radiology,

University Hospital, Philipps-University,

35033 Baldingerstrasse, Marburg, Germany

e-mail: mahnken@med.uni-marburg.de

C. Spreafico

Department of Interventional Radiology,

Fondazione IRCCS Istituto Nazionale dei Tumori di Milano, via Venezian 1, 20133 Milan, Italy

G. Maleux

Department of Radiology, University Hospitals Leuven,

Herestraat 49, 3000 Leuven, Belgium

T. Helmberger

Department of Diagnostic and Interventional Radiology,

Klinikum Bogenhausen, Englschalkinger Straße 77,

81925 Munich, Germany

T. F. Jakobs

Department of Diagnostic and Interventional Radiology,

Krankenhaus Barmherzige Brüder, Romanstraße 93,

80639 Munich, Germany
}

increasingly used in metastatic liver disease. Radiationbased approaches are appealing because they are known to be cytocidal in sufficient doses and they are independent from chemical or other energy-based treatment techniques. Although delivery of $>70 \mathrm{~Gy}$ is thought to be needed to achieve solid tumor destruction [3], the tolerance of normal liver tissue is approximately $30 \mathrm{~Gy}$ [4]. These conditions resulted in the idea of selective transarterial radiation delivery and triggered the development of various transarterial radiation-based therapies, with ${ }^{90} \mathrm{Y}$ microspheres being the current mainstay of radioembolization (RE).

History of ${ }^{90} \mathrm{Y}$ RE

${ }^{90} \mathrm{Y}$ RE was first reported in 1963 for embolizing prostate glands in dogs. Clinical reports from the early days of RE reported its use in lung and bone tumors [5, 6]. First patient series with intravenous application had poor outcomes [7]. Starting from the late 1970s, initial clinical series on nonselective intra-arterial ${ }^{90} \mathrm{Y}$ RE administered in the proper hepatic artery reported promising results, and it was observed that hypervascularized tumors were more likely to benefit from this type of therapy [8]. Several doseescalation studies in animals and humans followed these early reports [9], with an initial study indicating an excellent safety profile if angiographic findings and extrahepatic shunting were assessed before treatment [10].

\section{Definitions}

Complications (minor/major)

A minor complication is a treatment-related adverse event requiring no or minimal therapy with or without overnight 
hospitalization for observation. A major complication is a treatment-related adverse event requiring further therapy, increased level of care, or prolonged hospitalization [11].

\section{Lung Shunting Fraction (LSF)}

LSF is the percentage shunt fraction of radioactive-labeled microspheres from liver to lung.

\section{Postradioemblization Syndrome (PRS)}

PRS is any constellation of symptoms, including fatigue, low-grade fever, nausea and vomiting, abdominal discomfort, and malaise occurring shortly after RE.

\section{Radiation-Induced Liver Disease (RILD)}

RILD comprises mostly anicteric, nonmalignant ascites and elevation of alkaline phosphatase level of at least twice the upper normal level within 4 months after treatment.

\section{Technical Success}

Technical success is the ability to access the appropriate segments of the liver and to perform the treatment according to protocol.

\section{Pretreatment Imaging}

Before RE, some kind of whole body imaging with particular focus on the liver needs to be performed for local (liver) staging, assessment of hepatic vasculature, and evaluation of potential extrahepatic tumor spread. In addition, at least dual-phase contrast-enhanced computed tomography (CT) or dynamic contrast-enhanced magnetic resonance (MR) imaging of the liver is recommended. For tumors with a high glucose metabolism rate, like metastases from CRC, whole body ${ }^{18} \mathrm{~F}$ fluorodeoxyglucosepositron emission tomography-CT $\left({ }^{18} \mathrm{FDG}-\mathrm{PET} / \mathrm{CT}\right)$ can be also very helpful. Ultrasound, including contrastenhanced ultrasound, is only of limited use as a baseline modality in typically multifocal or even diffuse disease.

\section{Indications for Treatment and Contraindications}

Indications

Appropriateness of RE as a therapy in a particular case needs to be determined in a multidisciplinary tumor board. Patients with liver-dominant metastatic disease that is considered nonresectable by a liver surgeon or that is inoperable as a result of comorbidities are potential candidates for RE. In case of extrahepatic disease, the hepatic tumor burden needs to be considered the life-limiting element of disease. Good candidates have a life expectancy of more than 3 months, ideally with an Eastern Cooperative Oncology Group (ECOG) status score of $\leq 2$.

Typical indications for RE in metastatic colorectal liver disease include RE alone after failure of second-line chemotherapy, RE as salvage therapy, or RE as an adjuvant treatment to first- or second-line chemotherapy within a clinical trial. A neoadjuvant indication before resection may also be considered [12]. It may be suited as a salvage therapy in any other type metastatic liver disease. With several studies under way, the indications for RE in metastatic liver disease are evolving rapidly.

In hepatocellular carcinoma (HCC) patients, RE may be performed instead of transarterial chemoembolization (TACE) in patients fulfilling the criteria for TACE according to the Barcelona Clinic for Liver Cancer (BCLC) criteria. Because there are no randomized trials published comparing TACE and RE, in many institutions, RE is reserved for those patients whose disease either failed to respond to TACE, patients who present with (segmental or main stem) portal vein thrombosis (PVT), or patients with multifocal or diffuse disease.

\section{Contraindications}

There are only a few absolute contraindications for RE, including insufficient functional liver reserve (commonly used thresholds are total bilirubin $>2.0 \mathrm{mg} / \mathrm{dL}$ and albu$\min <3 \mathrm{~g} / \mathrm{dL}$ ), severe lung shunting resulting in a lung dose of $\geq 30$ Gy or anticipated nontarget embolization to the gastrointestinal tract that cannot be resolved by embolization techniques [13], and treatment with capecitabine within 2 months before RE with resin spheres.

Beside the typical relative contraindications for vascular procedures, such as uncorrectable coagulopathy, PVT is considered a relative contraindication with resin spheres, while the use of glass spheres is not limited by PVT. Initial results indicate that this technique is safe for treating HCC [14].

\section{Patient Preparation}

A thorough clinical assessment is mandatory, including ECOG status and/or Karnofsky index. A baseline blood sampling is performed with a focus on liver function tests and tumor markers, such as $\alpha$-fetoprotein and/or carcinoembryonic antigen. In addition, a comprehensive blood count, including platelets, coagulation panels, and renal and thyroid function, are obtained. 
In cases of impaired renal function, patients should be treated according to the current European Society of Urogenital Radiology guidelines [15]. Patients with known allergic reactions to contrast material should be prepared according to international guidelines [16]. The platelet count should be $>50,000$ and international normalized ratio should be $<1.5$; otherwise, appropriate measures to correct the coagulation state have to be undertaken. Peripheral i.v. access must be obtained, and the urinary bladder should be emptied before the initiation of the procedure.

Biliary obstructions need to be corrected. However, any impairment of the ampulla predisposes the patient to ascending infection. In these patients, preinterventional antibiotic therapy (e.g., ciprofloxacin, cotrimoxazole), ideally starting the day before the procedure, is strongly recommended, and long-term antibiotic treatment needs to be considered.

In view of the high variability of the hepatic arterial bed and the relevance of vascular anatomy, as described in Shepherd et al. [10], a detailed angiographic evaluation of the splanchnic arteries needs to be performed to identify tumor feeding vessels and, more importantly, hepatofugal vessels [17]. Considering the potentially deleterious effect of a dystopic spread of radioactive microspheres into extrahepatic sites such as the stomach, duodenum, or pancreas, it is important to ensure that there is no hepatofugal flow. This either requires embolization of vessels such as the gastroduodenal artery, right gastric artery, pancreaticoduodenal branches, and falciform artery or placement of the infusion catheter distally to all vessels with hepatofugal flow. The latter approach may be more liberally used with glass spheres, as reflux has never been reported to be a clinically relevant problem with this type of particles. However, with embolization being safer, this should be the preferred approach. These occluded vessels can revascularize, or collaterals can form around the embolized vessels. Therefore, the angiograms obtained during catheter positioning before radioactive particles are administered needs to thoroughly analyzed to exclude such revascularization.

Another particular feature of tumorous vessels is arteriovenous shunting, which allows microspheres to directly enter the venous system. Consequently, there is a certain degree of lung shunting, which causes microspheres to directly reach the lung, potentially causing radiation pneumonitis. With the highest tolerable dose of the lung being approximately 30 Gy for a single application, LSF must be assessed before treatment. For this purpose, diagnostic angiography and coiling is followed by infusion of approximately $200 \mathrm{MBq}$ technetium-99 m-macroaggregated albumin ( ${ }^{99 \mathrm{~m}}$ Tc-MAA) in the hepatic arteries, which are considered to supply the tumor-bearing liver territory.
Thereafter, the LSF is determined mostly from planar imaging. In case the LSF exceeds $10 \%$, the dose of the radioactive microspheres is adapted to the LSF to avoid radiation pneumonitis [18]. In case of a more than $20 \%$ LSF, RE with resin spheres should be omitted. With glass spheres, RE should be omitted if the injected activity shunted to the lungs exceeds $0.61 \mathrm{GBq}$. The dose of radioactive spheres may also be reduced by about $30 \%$ in case of impaired liver function [13]. It also is important to correlate the ${ }^{99 \mathrm{~m}}$ Tc-MAA scan with angiographic findings in order to identify potential vessels causing extrahepatic hot spots. Considering the complexity of the anatomy of the upper abdomen, the use of cross-sectional imaging (i.e., single-photon emission computed tomography [SPECT], SPECT/CT) is recommended to avoid superposition and to improve sensitivity for extrahepatic accumulations [19].

\section{Equipment Specifications}

In order to perform RE, a dedicated digital subtraction angiography $\mathrm{C}$-arm unit with a large detector providing high-quality imaging and sufficient magnification is indispensable. The availability of C-arm CT might be helpful in selected patients. Before the procedure, the area where it is anticipated that patient and/or staff may come into contact with radioactive material should be sealed with absorbent pads on the floor. The room and staff should be surveyed at the end of the procedure before leaving the floor pad. All potentially contaminated materials must be placed in a dedicated storage container.

Standard materials include the following: 4-5F catheters, typically with Cobra or Sidewinder configuration and 0.035-inch guide wires; $2.4-3 \mathrm{~F}$ microcatheters and dedicated 0.14- to 0.21-inch guide wires (essential for negotiating the target vessel); and embolic materials, including a variety of $2-8 \mathrm{~mm}$ coils and/or vascular plugs (for occluding vessels with hepatofugal flow). In some situations, detachable coils may be advantageous to avoid nontarget embolization. Foreign-body retrieval devices (microsnares), spasmolytic agents (e.g., nimodipine), and thrombolytic agents are needed to manage complications.

\section{Procedural Features and Variations of the Technique}

$\mathrm{RE}$ is a two-step procedure consisting of a diagnostic angiography followed by infusion of about $200 \mathrm{MBq}$ ${ }^{99} \mathrm{~m}$ Tc-MAA and the therapeutic injection of ${ }^{90} \mathrm{Y}$ microspheres. Both steps are performed in different sessions several days apart.

Sterile skin preparation of both groins is advisable. The site and direction of the arterial access (retrograde femoral 
or left brachial) depends on the preference of the interventionalist, although the femoral approach should be preferred because it is less prone to complications.

For diagnostic angiography, coiling, and ${ }^{99 \mathrm{~m}} \mathrm{Tc}-\mathrm{MAA}$ injection, after arterial puncture and placement of an arterial sheath, a $4 \mathrm{~F}$ or $5 \mathrm{~F}$ catheter is sequentially placed in the celiac trunk and the superior mesenteric artery, and digital subtraction angiograms are obtained. Baseline angiograms should be obtained in p.a. and RAO orientation. The use of a power injector for contrast injection is advisable.

Any accessory vessel needs to be identified.

In case of flow-obstructing lesions of the mesenteric or celiac inflow vessels, these areas should be treated before injection of any radioactive particles.

All hepatofugal vessels need to be superselectively embolized before the ${ }^{99 \mathrm{~m}}$ Tc-MAA injection, usually via microcatheters. These vessels typically include the gastroduodenal artery and the right gastric artery. In some patients, additional hepatofugal vessels arise from the intrahepatic vasculature, which needs to be occluded. Particular interest has to be paid to the left hepatic artery, where most hepatofugal vessels arise. The cystic artery may also be embolized before RE, but there is no consensus on the need to embolize this particular vessel. In addition, any arteriovenous shunt needs to be embolized.

Alternatively, the microcatheter for the ${ }^{99 \mathrm{~m}} \mathrm{Tc}-\mathrm{MAA}$ injection may be placed distal to the gastroduodenal artery.

An angiogram with the microcatheter in position for ${ }^{99 \mathrm{~m}}$ Tc-MAA needs to be performed and stored, as RE should be performed from the identical catheter position.

$\mathrm{RE}$ requires calculation, as the therapeutic activity differs between resin and glass spheres. In glass spheres, it is based on the nominal target dose and the patient's liver volume. In resin spheres, the body surface area, tumor volume, and liver volume are needed for calculating therapeutic activity. According to the instructions for use, an empiric method is also acceptable in resin spheres, but it is considered less safe than the body surface area method and cannot be recommended [13].

Vessel puncture and catheter placement are performed as described above.

Digital subtraction angiography of the target vessels is performed to exclude revascularization of previously embolized vessels or other vessels with hepatofugal flow. Untreated or revascularized hepatofugal vessels must be embolized before treatment. If in doubt, repeat ${ }^{99 \mathrm{~m}} \mathrm{Tc}$ MAA injection should be considered.

The tip of the microcatheter is placed on the identical position as during the ${ }^{99 \mathrm{~m}} \mathrm{Tc}-\mathrm{MAA}$ injection.

The infusion technique differs significantly between resin and glass spheres, as the spheres have different characteristics (Table 1). In glass spheres, far fewer spheres are injected compared to resin spheres. Because the injection is performed rapidly and without angiographic control, unobstructed flow without vasospasm or retrograde flow is needed. The flow of the spheres should mimic the hepatic artery flow, which is ensured by a visual inspection of a test injection of contrast agent without spheres. The injection itself is typically completed within $5 \mathrm{~min}$. As far more resin spheres are infused, subsequent changes in blood flow characteristics may occur. Thus, regular controls are needed to ensure an unobstructed flow. The infusion of resin spheres takes about $30-40 \mathrm{~min}$ and is completed if either the total intended dose has been administered or if there is complete stasis before the entire intended dose has been infused.

During the injection, the interventionalist should avoid any contact with the catheter because this goes along with very high focal radiation exposure.

All materials are then carefully removed; the interventionalist needs to avoid spilling any fluid that was in contact with the catheters. All materials are collected in a dedicated container.

Although RE traditionally started as a whole liver treatment via the surgically exposed gastroduodenal artery, the interventional approach easily permits a lobar treatment (i.e., sequential separate treatment of the left and right liver lobes) at an interval of several weeks. The latter appears to be safer, with a lower rate of RILD [20].

\section{Medication and Periprocedural Care}

During the procedure, routine monitoring of vital function is mandatory. ECG monitoring, pulse oximetry, and repeated blood pressure measurements are strongly recommended.

Although not mandatory, some premedication is recommended to enhance tolerance to treatment. This includes gastrointestinal ulcer prophylaxis (proton pump inhibitor or H2-blockers initiated 1 week before RE and continued for at least 4 weeks after treatment), as well as nausea prophylaxis with antiemetics such as ondansetron. PRS may be alleviated by steroids. Thus, oral treatment with corticosteroids (e.g., dexamethasone $4 \mathrm{mg}$ ) is recommended for 3 days starting the day of the procedure. An additional intravenous high-dose administration (e.g., prednisolone $250 \mathrm{mg}$ ) directly before the treatment is considered helpful for tolerance.

Embolization, particularly with resin spheres, may be painful, and slow infusion of potent i.v. analgesia such as pethidine should be considered. In very few patients, oral analgesia may be required for up to 1 week after the procedure. 
Table 1 Characteristics of commercially available ${ }^{90} \mathrm{Y}$ particles

FDA U.S. Food and Drug Administration, $C R C$ colorectal cancer

\begin{tabular}{lll}
\hline Feature & SIR-sphere & TheraSphere \\
\hline Isotope & ${ }^{90} \mathrm{Y}$ & $90 \mathrm{Y}$ \\
Half-life $(\mathrm{h})$ & 64.2 & 64.2 \\
Material & Resin & Glass \\
Diameter $(\mu \mathrm{m})$ & $20-60$ & $20-30$ \\
Activity per particle $(\mathrm{Bq})$ & 50 & 2500 \\
Spheres per 3 GBq & $40-80 \times 10^{6}$ & $1.2 \times 10^{6}$ \\
Specific gravity $(\mathrm{g} / \mathrm{ml})$ & 1.6 & 3.2 \\
Embolic effect & Mild & Negligible \\
Contrast injection & During infusion & No \\
FDA-approved indication & CRC liver metastases with intrahepatic floxuridine & HCC \\
\hline
\end{tabular}

\section{Postprocedural Follow-up Care (Including Imaging)}

A thorough posttreatment assessment is mandatory including laboratory and imaging follow-up in order to monitor treatment response as well as toxicity. Laboratory tests include all parameters of liver function and tumor markers. Contrast-enhanced CT, MRI, or PET/CT should be performed every 3 months after the procedure to assess response to treatment. The posttreatment assessment should follow the research reporting standards for RE, with treatment response on imaging being accessed by Response Evaluation Criteria in Solid Tumors (RECIST) 1.1 [21]. However, often the lesion will not change significantly in size despite effective treatment. Therefore, other response criteria are needed, incorporating additional information. Lack of contrast enhancement is such a parameter. For $\mathrm{HCC}$, this is already part of the recently published modified RECIST, where complete response corresponds to the disappearance of any intratumoral arterial enhancement and partial response is defined as a minimum of $30 \%$ decrease in the sum of diameters of viable (i.e., arterial phase contrast enhancing) target lesions [22]. Besides these established criteria, functional parameters derived from PET/CT such as total lesion glycolysis will become of increasing interest, as they have been demonstrated to be strongly correlated with prognosis [23].

\section{Outcome}

\section{Effectiveness}

\section{Hepatocellular Carcinoma}

A key study to support the efficacy of selective internal radiation treatment in the treatment of $\mathrm{HCC}$ has been published by Vente et al. [24]. This meta-analysis included 14 studies with a total of 425 patients. Twelve studies presented data of tumor response for a total of 318 patients.
Treatment with resin microspheres was associated with a significantly higher proportion of patients with any response than patients being treated with glass microspheres ( 0.89 vs. $0.78, p=0.02)$. Median survival was reported by seven studies and varied between 7.1 and 21 months from the point of treatment and 9.4-24 months from diagnosis or detection of tumor recurrence. Neither stratification according to the BCLC staging and treatment algorithm nor the Child-Pugh class was provided.

There are several case series and single-arm trials on the use of RE in HCC. In a retrospective study, Salem and colleagues [25] reported on 291 patients with nonresectable HCC that was treated with glass microspheres. According to the European Association for the Study of Liver (EASL) criteria, the overall response rate was $57 \%$. The disease of $12 \%$ of the patients was successfully downstaged so patients could receive curative therapies such as resection or transplantation. The reported median time to progression was 7.9 months. When stratified according to the BCLC system, the overall survival was $26.9,17.2$, and 7.3 and 5.4 months in BCLC stage A, B, and C patients without or with extrahepatic disease, respectively. With regard to the Child-Pugh category, overall survival was 17.2/8.7 and 7.7/ 3.2 for Child-Pugh A and B patients without and with extrahepatic metastases, respectively.

In a large retrospective multicentre study of RE with resin microspheres, Sangro et al. [26] presented the results of 325 patients with nonresectable HCC. In BCLC stage A, $\mathrm{B}$, and $\mathrm{C}$ patients, overall survival was $24.4,16.9$, and 10 months, respectively. Interestingly, overall survival in patients with BCLC stage $\mathrm{C}$ disease was neither significantly affected by the presence of PVT (10.2 vs. 9.3 months; $p=0.826$ ), ECOG performance status $1-2$ (9.9 vs. 10.8 months; $p=0.844$ ), or extrahepatic metastases (7.4 vs. 10.2 months; $p=0.137$ ).

Hilgard et al. published a prospective phase II study of RE with glass microspheres in 108 patients with nonresectable HCC [27]. According to EASL criteria, complete responses were determined in $3 \%$ of patients, partial 
responses in $37 \%$, stable disease $53 \%$, and primary progression in $6 \%$ of patients. Time to progression was 10.0 months, whereas the median overall survival was 16.4 months in BCLC stage A or B patients, and was not reached in BCLC stage $C$ patients.

A prospective phase II study comparing RE with glass microspheres in patients with $(n=37)$ or without $(n=71)$ PVT was published by Kulik et al. [28]. There were no significant differences between the two cohorts in noncirrhotic patients, while cirrhotic patients with PVT had a higher tumor burden ( $>50 \%$ liver burden: 33 vs. $4 \%$; $p<0.001$ ), elevated baseline bilirubin $>2 \mathrm{mg} / \mathrm{dL}$ (23 vs. $6 \%$ ), and portal hypertension ( 87 vs. $62 \% ; p=0.016$ ). The adverse event rate was higher only in patients with both main stem PVT and cirrhosis. The overall survival was shorter in patients with main stem PVT compared to branch PVT or without PVT (median 4.4 vs. 10.0 vs. 15.4 months; $p=0.0052$ ) and also varied depending on the presence of cirrhosis.

There are only few comparative studies of TACE and RE. A retrospective cohort comparison of RE (resin or glass microspheres, $n=38$ ) vs. TACE (conventional TACE/drug-eluting bead TACE, $n=35$ ) was published by Lance et al. [29]. There were no significant differences between the two cohorts and no significant difference in overall survival between RE and TACE (median 8.0 vs. 10.3 months) cohorts ( $p=0$ 0.33). However, postembolization syndrome was significantly more severe in patients receiving TACE, which led to increased total hospitalization rates in these patients (0.5 vs. 3.5 days; $p<0.001)$. Accordingly, RE was associated with fewer adverse events than TACE (10 vs. $17 ; p=0.05$ ) and lower 30-day mortality ( 0 vs. $3 ; p=0.07$ ). As a limitation of this study, the TACE protocol was not standardized, and different chemotherapeutic agents as well as different embolic materials were used.

In a retrospective matched-cohort comparison of RE vs. standard therapy or best supportive care (in patients who were either treated before adoption of RE or who were technically contraindicated for $\mathrm{RE}$ as a result of lung or gastrointestinal shunting), D'Avola et al. [30] proved the efficacy of RE with prolonged overall survival when compared to standard therapy/best supportive care (median 16 vs. 8 months; $p<0.001$ ).

Salem et al. [31] recently reported on a retrospective cohort comparison of RE with glass microspheres $(n=122)$ vs. conventional TACE $(n=123)$ in patients with nonresectable HCC. RE patients were older than TACE patients (median 66 vs. 61 years; $p<0.001$ ) and trended toward having larger tumors (median largest 4.5 vs. $3.6 \mathrm{~cm} ; p=0.1)$. RE patients reported less abdominal pain (15 vs. $38 \% ; p<0.001)$; otherwise, there were no other differences in toxicities detected. Overall response rate for RE and TACE (respectively, World Health Organization criteria 49 vs. $36 \%, p=0.052$; and EASL criteria 72 vs. $69 \%, p=0.748$ ) did not differ significantly, but RE significantly prolonged time to progression compared to conventional TACE (13.3 vs. 8.4 months; $p=0.046)$. There was no significant difference in overall survival between RE and TACE (20.5 vs. 17.4 months; $p=232)$, apart from a small number of BCLC stage C patients (22.1 vs. 9.3 months; $p=0.04$ ).

\section{Metastases}

Corresponding to U.S. Food and Drug Administration approval, most data are available for the treatment of CRC liver metastases with resin microspheres, but there are also data that use glass microspheres for treating metastatic liver disease (Table 2). There also is a substantial amount of data on RE in liver metastases from neuroendocrine tumors, breast cancer, and uveal melanoma (Table 3). Currently available data are quite heterogeneous, with most data being obtained in a salvage situation with or without additional use of systemic chemotherapy.

There is one meta-analysis on the effects of RE in liver metastases from CRC. The review comprises 681 patients from 18 studies. The meta-analysis covers first-line $(n=195)$ as well as salvage therapy $(n=465)$ in combination with various cytostatic agents. Response to treatment was independent from the chemotherapeutic agent. The estimated response based on a regression model was better in the first-line setting (0.91) when compared with the salvage setting (0.79). Median survival after the diagnosis of metastatic CRC and ${ }^{90} \mathrm{Y}$ RE varied from 6.7 to 17.0 months and 10.8-29.4 months, respectively [24]. Unfortunately, the quality of the present data does not permit reliable analysis of overall survival.

There are only few randomized controlled trials (RCT), all of them on the treatment of liver metastases from CRC. In 2001 Gray et al. reported data on the use of hepatic artery chemotherapy (HAC) with floxuridine with or without additional RE. The median time to hepatic disease progression was significantly longer in the group receiving additional RE (9.7 vs. 15.9 months, $p=0.001$ ). Overall survival was also favorable for the combined treatment group with 1-, 2-, and 3-year survival of 72, 39, and 17 in the HAC plus RE group compared to 68,29 , and $6.5 \%$ for HAC alone [32]. In 2004 the same group reported favorable data from a small RCT on the use of systemic fluorouracil/leucovorin chemotherapy with or without an additional single administration of ${ }^{90} \mathrm{Y}$ resin microspheres in patients with advanced CRC liver metastases. In this small study with only 21 patients, time to progression (18.6 vs. 3.6 months, $p<0.0005)$ and median survival (29.4 vs. 12.8 months, $p=0.02$ ) were significantly longer for 
patients receiving the combined treatment [33]. The most recent RCT by Hendlisz et al. compared the combination of $\mathrm{RE}$ with fluorouracil vs. protracted intravenous infusion of fluorouracil alone in 46 patients with nonresectable, chemotherapy-refractory, liver-limited metastatic CRC. Time to any tumor progression as well as time to hepatic tumor progression were favorable for the combination therapy, at 2.1 months vs. $4.5(p=0.03)$ and 5.5 months $(p=0.003)$, respectively, keeping in mind that crossover from the fluorouracil arm into the RE arm was permitted. Median overall survival was favorable for the combination treatment but failed to demonstrate a significant advantage (10.0 vs. 7.3 months; $p=0.80$ ) [34].

So far, there is only one study comparing chemoembolization vs. ${ }^{90} \mathrm{Y}$ RE for liver-dominant metastatic CRC as salvage therapy [35]. This retrospective study comprised 36 patients (21 chemoembolization; 37 procedures vs. 15 ${ }^{90} \mathrm{Y}$ RE). There were no outcome differences, with a median survival of 7.7 months for chemoembolization and 6.9 months for RE $(p=0.27)$. Because of the inhomogeneity of study protocols, comparison of studies on RE with historic cohorts that underwent chemoembolization is not reasonably possible.

\section{Complications and Their Management}

\section{Toxicity and Complications}

$\mathrm{RE}$ is a safe procedure with a lower toxicity than chemoembolization [29, 31]. The most common adverse effect is PRS, which occurs in up to $50 \%$ of the patients and may last for about 2 weeks. A transient elevation of liver function tests, specifically an increase in alkaline phosphatase, bilirubin, and alanine transferase, have to be considered as a normal toxicity of the treatment. There are several specific complications of RE, which may be separated in intra- and extrahepatic complications, including radiation-induced pneumonitis, gastrointestinal ulcerations, pancreatitis, RILD, portal hypertension, cholecystitis, and bile duct injuries [36]. Extrahepatic complications occur if relevant amounts of radioactive particles inadvertently spread to other organs than the liver. Radiation-induced pneumonitis is a restrictive ventilatory dysfunction; it may occur in patients with a LSF of about $15 \%$ or more. A single dose of $\geq 30$ Gy to the lungs may result in pulmonary toxicity. It occurs in less of $1 \%$ of patients if standard dosimetry is performed [37]. Gastrointestinal ulcerations and pancreatitis occur in less than $5 \%$ of patients after meticulous patient preparation [38]. RILD eventually occurs in up to $4 \%$ of patients, and it is more common in patients with preexisting liver dysfunction [39].

\section{Management of Complications}

Management of complications and adverse effects is mostly symptomatic. PRS is treated symptomatically. RILD and radiation-induced pneumonitis are also treated with symptomatic measures supported by high-dose steroids in an attempt to decrease inflammation. Portal hypertension is also treated if symptomatic, with a particular focus on endoscopic treatment of bleeding complications. Biliary sequelae are only treated if symptomatic. Radiation-induced cholecystitis requiring cholecystectomy occurs in less than $1 \%$ of patients. Gastrointestinal ulcerations are only treated surgically if medical and endoscopic treatment fail.

Table 2 Summary of studies on RE in liver metastases from CRC with more than 50 patients

\begin{tabular}{|c|c|c|c|c|c|c|c|c|c|c|}
\hline Study & $\begin{array}{l}\text { Patient/ } \\
\text { response data }\end{array}$ & $\begin{array}{l}\text { Particle } \\
\text { type }\end{array}$ & $\begin{array}{l}\text { Additional therapy } \\
\text { (per protocol) }\end{array}$ & Setting & Design & CR & PR & SD & PD & $\begin{array}{l}\text { Median survival from } \\
\text { RE (mo) }\end{array}$ \\
\hline $\begin{array}{l}\text { Kennedy } \\
\text { [40] }\end{array}$ & $208 / 208$ & Resin & & Salvage & Retrospective & 0 & 36 & 55 & 10 & $\begin{array}{l}\text { Response, } 10.5 ; \\
\text { nonresponse, } 4.5\end{array}$ \\
\hline $\begin{array}{c}\text { Stubbs } \\
\text { [41] }\end{array}$ & $100 / 80$ & Resin & $\mathrm{HAI}+5-\mathrm{FU}$ & Mixed & Retrospective & 1 & 73 & 20 & 6 & 11 \\
\hline Sato [42] & $51 / 51$ & Glass & & Salvage & Prospective & NA & NA & NA & NA & 15.2 \\
\hline $\begin{array}{l}\text { Mulcahy } \\
\text { [43] }\end{array}$ & $72 / 72$ & Glass & & Mixed & Prospective & 0 & 40 & 45 & 15 & 14.5 \\
\hline $\begin{array}{l}\text { Cosimelli } \\
{[44]}\end{array}$ & $50 / 46$ & Resin & & Salvage & Prospective & 2 & 24 & 26 & 48 & 12.6 \\
\hline $\begin{array}{r}\text { Evans } \\
{[45]}\end{array}$ & 140/NA & Resin & & $\begin{array}{l}\text { Salvage }>2 \text { nd } \\
\text { line }\end{array}$ & Retrospective & NA & NA & NA & NA & 7.9 \\
\hline Chua [46] & $140 / 140$ & Resin & & Mixed & Retrospective & 1 & 31 & 31 & 37 & 9 \\
\hline
\end{tabular}

$\overline{R E}$ radioembolization, $C R C$ colorectal cancer, $H A I$ hepatic artery infusion, 5-fluorouracil, $R C T$ randomized controlled trial, $N A$ not available, $C R$ complete response, $P R$ partial response, $S D$ stable disease, $A R$ any response, $P D$ progressive disease 
Table 3 Summary of studies on RE in liver metastases of NET, breast cancer and uveal melanoma with more than 25 patients

\begin{tabular}{|c|c|c|c|c|c|c|c|c|c|c|}
\hline Study & $\begin{array}{l}\text { Patient/response } \\
\text { data }\end{array}$ & Particle type & Entity & Setting & Design & $\mathrm{CR}$ & PR & SD & PD & $\begin{array}{l}\text { Median } \\
\text { survival } \\
\text { from RE (mo) }\end{array}$ \\
\hline Rhee [47] & $42 / 29$ & Both & NET & Salvage & Prospective & 0 & 51.7 & 41.4 & 6.9 & 25 \\
\hline Kennedy [48] & $148 / 168$ & Resin & NET & NA & Retrospective & 3 & 66.7 & 25 & 5.3 & 70 \\
\hline King [49] & $34 / 34$ & Resin & NET & Salvage & Prospective & 14.7 & 35.3 & 14.7 & 35.3 & 27.6 \\
\hline Saxena [50] & $48 / 48$ & Resin & NET & Salvage & Retrospective & 15 & 40 & 23 & 22 & 35 \\
\hline Cao [51] & $58 / 51$ & Resin & NET & Mixed & Retrospective & 11.7 & 27.5 & 27.5 & 33.3 & 36 \\
\hline Paprottka [52] & $42 / 42$ & Resin & NET & NA & Retrospective & 0 & 22.5 & 75 & 2.5 & NA \\
\hline Jakobs [53] & $30 / 23$ & Resin & Breast & Salvage & Retrospective & 0 & 61 & 35 & 4 & 11.7 \\
\hline Coldwell [54] & $44 / 36$ & Resin & Breast & Salvage & Retrospective & 0 & 47 & 47 & 6 & NA \\
\hline Bangash [55] & $27 / 23$ & Glass & Breast & Salvage & Prospective & NA & 39.1 & 52.1 & 8.8 & NA \\
\hline Gonsalves [56] & $32 / 32$ & Resin & Uveal melanoma & Salvage & Retrospective & 3 & 3 & 56 & 38 & 10 \\
\hline
\end{tabular}

$R E$ radioembolization, $N E T$ neuroendocrine tumors, $N A$ not available, $C R$ complete response, $P R$ partial response, $S D$ stable disease, $P D$ progressive disease

\section{Conclusions}

$\mathrm{RE}$ is a powerful way to achieve local tumor response and improve survival in advanced stages of primary and secondary liver malignancies. In metastatic disease, it has demonstrated its potential in salvage situations, and initial data in neoadjuvant settings are encouraging. Its future use in earlier stages of disease is the subject of ongoing clinical trials. In HCC, RE is particularly useful in patients with nonresectable/nonablatable HCC, either as an alternative to TACE, or in patients who are not suitable for TACE or whose disease has failed to respond to TACE. With careful patient selection and meticulous pretreatment assessment, this modality has an excellent safety profile.

Conflict of interest Tobias Jacob is a consultant for SIRTeX Medical Europe and has received lecture fees from SIRTeX Medical Europe and Siemens Healthcare. Andreas Mahnken received fees for developing educational presentations from Bayer Healthcare. The other authors declare that they have no conflict of interest.

\section{References}

1. Jemal A, Bray F, Center MM et al (2011) Global cancer statistics. CA Cancer J Clin 61:69-90

2. Davies JM, Goldberg RM (2011) Treatment of metastatic colorectal cancer. Semin Oncol 38:552-560

3. Dawson LA, McGinn CJ, Normolle D et al (2000) Escalated focal liver radiation and concurrent hepatic artery fluorodeoxyuridine for unresectable intrahepatic malignancies. J Clin Oncol 18:2210-2218

4. Dawson LA, Normolle D, Balter JM et al (2002) Analysis of radiation-induced liver disease using the Lyman NTCP model. Int J Radiat Oncol Biol Phys 53:810-821

5. Ariel IM, Pack GT (1966) The treatment of cancer metastases in the lung by means of radiating microspheres. Thoraxchir Vask Chir 14:286-307
6. Simon N, Siffert R, Baron MG et al (1968) Preoperative irradiation of osteogenic sarcoma with intra-arterially injected yttrium90 microspheres. Case report. Cancer 21:453-455

7. Blanchard RJ, LaFave JW, Kim YS (1965) Treatment of patients with advanced cancer using Y-90 microspheres. Cancer 18: 375-380

8. Mantravadi RV, Spigos DG, Tan WS et al (1982) Intraarterial yttrium 90 in the treatment of hepatic malignancy. Radiology $142: 783-786$

9. Wollner I, Knutsen C, Smith P et al (1988) Effects of hepatic arterial yttrium 90 glass microspheres in dogs. Cancer 61: 1336-1344

10. Shepherd FA, Rotstein LE, Houle S et al (1992) A phase I dose escalation trial of yttrium-90 microspheres in the treatment of primary hepatocellular carcinoma. Cancer 70:2250-2254

11. Sacks D, McClenny TE, Cardella JF, Lewis CA (2003) Society of Interventional Radiology clinical practice guidelines. J Vasc Interv Radiol 14:S199-S202

12. Van den Eynde M, Flamen P, El Nakadi I et al (2008) Inducing resectability of chemotherapy refractory colorectal liver metastasis by radioembolization with yttrium- 90 microspheres. Clin Nucl Med 33:697-699

13. Kennedy A, Nag S, Salem R et al (2007) Recommendations for radioembolization of hepatic malignancies using yttrium-90 microsphere brachytherapy: a consensus panel report from the radioembolization brachytherapy oncology consortium. Int $\mathrm{J}$ Radiat Oncol Biol Phys 68:13-23

14. Iñarrairaegui $M$, Thurston KG, Bilbao JI et al (2010) Radioembolization with use of yttrium-90 resin microspheres in patients with hepatocellular carcinoma and portal vein thrombosis. J Vasc Interv Radiol 21:1205-1212

15. Stacul F, van der Molen AJ, Reimer P, Contrast Media Safety Committee of European Society of Urogenital Radiology (ESUR) et al (2011) Contrast induced nephropathy: updated ESUR Contrast Media Safety Committee guidelines. Eur Radiol 21:2527-2541

16. Bush WH Jr (1999) Treatment of acute contrast reactions. In: Bush WH Jr, Krecke KN, King BF Jr et al (eds) Radiology Life Support. Oxford University Press, New York, pp 31-51

17. Liu DM, Salem R, Bui JT et al (2005) Angiographic considerations in patients undergoing liver-directed therapy. J Vasc Interv Radiol 16:911-935

18. Wright CL, Werner JD, Tran JM et al (2012) Radiation pneumonitis following yttrium-90 radioembolization: case report and literature review. J Vasc Interv Radiol 23:669-674 
19. Ahmadzadehfar H, Sabet A, Biermann K et al (2010) The significance of ${ }^{99 \mathrm{~m}} \mathrm{Tc}-\mathrm{MAA}$ SPECT/CT liver perfusion imaging in treatment planning for ${ }^{90} \mathrm{Y}$-microsphere selective internal radiation treatment. J Nucl Med 51:1206-1212

20. Seidensticker R, Seidensticker M, Damm R et al (2012) Hepatic toxicity after radioembolization of the liver using (90)Y-microspheres: sequential lobar versus whole liver approach. Cardiovasc Intervent Radiol 35:1109-1118

21. Salem R, Lewandowski RJ, Gates VL, Technology Assessment Committee; Interventional Oncology Task Force of the Society of Interventional Radiology et al (2011) Research reporting standards for radioembolization of hepatic malignancies. J Vasc Interv Radiol 22:265-278

22. Lencioni R, Llovet JM (2010) Modified RECIST (mRECIST) assessment for hepatocellular carcinoma. Semin Liver Dis 30:52-60

23. Gulec SA, Suthar RR, Barot TC, Pennington K (2011) The prognostic value of functional tumor volume and total lesion glycolysis in patients with colorectal cancer liver metastases undergoing ${ }^{90} \mathrm{Y}$ selective internal radiation therapy plus chemotherapy. Eur J Nucl Med Mol Imaging 38:1289-1295

24. Vente MA, Wondergem M, van der Tweel I et al (2009) Yttrium90 microsphere radioembolization for the treatment of liver malignancies: a structured meta-analysis. Eur Radiol 19:951-959

25. Salem R, Lewandowski RJ, Mulcahy MF et al (2010) Radioembolization for hepatocellular carcinoma using yttrium-90 microspheres: a comprehensive report of long-term outcomes. Gastroenterology 138:52-64

26. Sangro B, Carpanese L, Cianni R, European Network on Radioembolization with Yttrium-90 Resin Microspheres (ENRY) et al (2011) Survival after yttrium-90 resin microsphere radioembolization of hepatocellular carcinoma across Barcelona clinic liver cancer stages: a European evaluation. Hepatology 54:868-878

27. Hilgard P, Hamami M, Fouly AE et al (2010) Radioembolization with yttrium-90 glass microspheres in hepatocellular carcinoma: European experience on safety and long-term survival. Hepatology 52:1741-1749

28. Kulik LM, Carr BI, Mulcahy MF et al (2008) Safety and efficacy of ${ }^{90} \mathrm{Y}$ radiotherapy for hepatocellular carcinoma with and without portal vein thrombosis. Hepatology 47:71-81

29. Lance C, McLennan G, Obuchowski N et al (2011) Comparative analysis of the safety and efficacy of transcatheter arterial chemoembolization and yttrium-90 radioembolization in patients with unresectable hepatocellular carcinoma. J Vasc Interv Radiol 22:1697-1705

30. D'Avola D, Lñarrairaegui M, Bilbao JI et al (2009) A retrospective comparative analysis of the effect of Y90-radioembolization on the survival of patients with unresectable hepatocellular carcinoma. Hepatogastroenterology 56:1683-1688

31. Salem R, Lewandowski RJ, Kulik L et al (2011) Radioembolization results in longer time-to-progression and reduced toxicity compared with chemoembolization in patients with hepatocellular carcinoma. Gastroenterology 140:497-507

32. Gray B, Van Hazel G, Hope M et al (2001) Randomised trial of SIR-Spheres plus chemotherapy vs. chemotherapy alone for treating patients with liver metastases from primary large bowel cancer. Ann Oncol 12:1711-1720

33. Van Hazel G, Blackwell A, Anderson J et al (2004) Randomised phase 2 trial of SIR-Spheres plus fluorouracil/leucovorin chemotherapy versus fluorouracil/leucovorin chemotherapy alone in advanced colorectal cancer. J Surg Oncol 88:78-85

34. Hendlisz A, Van den Eynde M, Peeters M et al (2010) Phase III trial comparing protracted intravenous fluorouracil infusion alone or with yttrium-90 resin microspheres radioembolization for liver-limited metastatic colorectal cancer refractory to standard chemotherapy. J Clin Oncol 28:3687-3694

35. Hong K, McBride JD, Georgiades CS et al (2009) Salvage therapy for liver-dominant colorectal metastatic adenocarcinoma: comparison between transcatheter arterial chemoembolization versus yttrium-90 radioembolization. J Vasc Interv Radiol 20:360-367

36. Riaz A, Lewandowski RJ, Kulik LM et al (2009) Complications following radioembolization with yttrium-90 microspheres: a comprehensive literature review. J Vasc Interv Radiol 20:1121-1130

37. Salem R, Parikh P, Atassi B et al (2008) Incidence of radiation pneumonitis after hepatic intra-arterial radiotherapy with yttrium90 microspheres assuming uniform lung distribution. Am J Clin Oncol 31:431-438

38. Murthy R, Brown DB, Salem R et al (2007) Gastrointestinal complications associated with hepatic arterial yttrium-90 microsphere therapy. J Vasc Interv Radiol 18:553-561

39. Kennedy AS, McNeillie P, Dezarn WA et al (2009) Treatment parameters and outcome in 680 treatments of internal radiation with resin (90)Y-microspheres for unresectable hepatic tumors. Int J Radiat Oncol Biol Phys 74:1494-1500

40. Kennedy AS, Coldwell D, Nutting C et al (2006) Resin ${ }^{90} \mathrm{Y}$ microsphere brachytherapy for unresectable colorectal liver metastases: modern USA experience. Int $\mathrm{J}$ Radiat Oncol Biol Phys 65:412-425

41. Stubbs RS, O'Brien I, Correia MM (2006) Selective internal radiation therapy with ${ }^{90} \mathrm{Y}$ microspheres for colorectal liver metastases: single-centre experience with 100 patients. ANZ J Surg 76:696-703

42. Sato KT, Lewandowski RJ, Mulcahy MF et al (2008) Unresectable chemorefractory liver metastases: radioembolization with ${ }^{90} \mathrm{Y}$ microspheres-safety, efficacy, and survival. Radiology 247:507-515

43. Mulcahy MF, Lewandowski RJ, Ibrahim SM et al (2009) Radioembolization of colorectal hepatic metastases using yttrium-90 microspheres. Cancer 115:1849-1858

44. Cosimelli M, Golfieri R, Cagol PP, Italian Society of Locoregional Therapies in Oncology (SITILO) et al (2010) Multi-centre phase II clinical trial of yttrium-90 resin microspheres alone in unresectable, chemotherapy refractory colorectal liver metastases. Br J Cancer 103:324-331

45. Evans KA, Richardson MG, Pavlakis N et al (2010) Survival outcomes of a salvage patient population after radioembolization of hepatic metastases with yttrium-90 microspheres. J Vasc Interv Radiol 21:1521-1526

46. Chua TC, Bester L, Saxena A, Morris DL (2011) Radioembolization and systemic chemotherapy improves response and survival for unresectable colorectal liver metastases. J Cancer Res Clin Oncol 137:865-873

47. Rhee TK, Lewandowski RJ, Liu DM et al (2008) ${ }^{90} \mathrm{Y}$ radioembolization for metastatic neuroendocrine liver tumors: preliminary results from a multi-institutional experience. Ann Surg 247:1029-1035

48. Kennedy AS, Dezarn WA, McNeillie P et al (2008) Radioembolization for unresectable neuroendocrine hepatic metastases using resin ${ }^{90} \mathrm{Y}$-microspheres: early results in 148 patients. Am J Clin Oncol 31:271-279

49. King J, Quinn R, Glenn DM et al (2008) Radioembolization with selective internal radiation microspheres for neuroendocrine liver metastases. Cancer 113:921-929

50. Saxena A, Chua TC, Bester L et al (2010) Factors predicting response and survival after yttrium-90 radioembolization of unresectable neuroendocrine tumor liver metastases: a critical appraisal of 48 cases. Ann Surg 251:910-916 
51. Cao CQ, Yan TD, Bester L et al (2010) Radioembolization with yttrium microspheres for neuroendocrine tumour liver metastases. Br J Surg 97:537-543

52. Paprottka PM, Hoffmann RT, Haug A et al (2012) Radioembolization of symptomatic, unresectable neuroendocrine hepatic metastases using yttrium-90 microspheres. Cardiovasc Intervent Radiol 35:334-342

53. Jakobs TF, Hoffmann RT, Fischer T et al (2008) Radioembolization in patients with hepatic metastases from breast cancer. J Vasc Interv Radiol 19:683-690
54. Coldwell DM, Kennedy AS, Nutting CW (2007) Use of yttrium-90 microspheres in the treatment of unresectable hepatic metastases from breast cancer. Int J Radiat Oncol Biol Phys 69:800-804

55. Bangash AK, Atassi B, Kaklamani V et al (2007) ${ }^{90} \mathrm{Y}$ radioembolization of metastatic breast cancer to the liver: toxicity, imaging response, survival. J Vasc Interv Radiol 18:621-628

56. Gonsalves CF, Eschelman DJ, Sullivan KL et al (2011) Radioembolization as salvage therapy for hepatic metastasis of uveal melanoma: a single-institution experience. AJR Am J Roentgenol 196:468-473 